\title{
UN ANÁLISIS DE SERIES DE TIEMPO MEDIANTE MODELOS SARIMAX PARA LA PROYECCIÓN DE DEMANDA DE CARGA EN EL PUERTO DEL CALLAO
}

\author{
Víctor Changa
}

\begin{abstract}
RESUMEN
El objetivo principal de este artículo es estimar y proporcionar modelos de pronóstico para predecir el rendimiento de carga para el Puerto del Callao del 2019 al 2023. Estos resultados podrían servir para hacer un análisis prospectivo, mejorar las decisiones de inversión y determinar las tarifas del puerto. Para este propósito, los modelos de series temporales SARIMAX se utilizan con la inclusión de entradas exógenas que son representativas del rendimiento de carga de las tres terminales del Puerto del Callao: APMTC, DPWC y TC. Los resultados del pronóstico indican que para el año 2023 se alcanzará un total de 17 millones de TM y 3.4 millones de TEU's, activando los gatillos de inversión para APMTC y DPWC.
\end{abstract}

JEL: C22; L91; R41

PALABRAS CLAVES: Modelos SARIMAX, Análisis de Series de Tiempo, Predicción de Flujos de Carga Marítima, Modelos de Predicción, Puerto del Callao

\begin{abstract}
The main objective of this article is to estimate and provide forecast models in order to predict cargo throughput for the Port of Callao from 2019 to 2023. These results could serve to make a prospective analysis, improve the decisions of investment and determine port rates. For this purpose, SARIMAX time series models are used with the inclusion of exogenous inputs which are representative of the cargo throughput of the three terminals of the Port of Callao: APMTC, DPWC and TC. The forecast results indicate that by the year 2023 a total of 17 million MT's and 3.4 million TEU's will be reached, activating investment triggers for APMTC and DPWC.
\end{abstract}

JEL: C22; L91; R41

KEYWORDS: SARIMAX models, Time Series Analysis, Forecasting of maritime cargo flows, Forecasting Models, Port of Callao.

A Investigador docente de la Escuela Profesional de Economía de la Facultad de Ciencias Contables Económicas y Financieras de la Universidad de San Martín de Porres y Analista de Estudios Económicos del Organismo Supervisor de la Inversión en Infraestructura de Transporte de Uso Público (OSITRAN). Se agradece enormemente la asistencia de investigación de Fernando Castro Muro quien ha realizado un eficiente trabajo. 


\section{INTRODUCCIÓN}

La demanda de transporte se caracteriza por ser una demanda derivada, debido a que esta por sí misma no se demanda como actividad final, sino que se encuentra asociado con la demanda de lo que se transporta, ya sea pasajeros o carga (Gines de Rus, 2003). La demanda de carga portuaria, no es la excepción ya que ésta se encuentra estrechamente relacionada con la demanda de los productos que se importan y aquellos que se exportan, es decir que depende de la actividad económica del país y del comercio internacional. De esta manera, cambios en los precios de estos productos como por ejemplo los commodities, así como mayor crecimiento económico del país y de los socios comerciales afectan el tráfico de carga en el sector portuario. Asimismo, mercados relacionados como el de infraestructura portuaria, el de las compañías navieras, así los operadores logísticos portuarios se ven afectados por la demanda de carga portuaria.

Así, la información sobre la demanda y la proyección de la misma resulta ser una variable de suma importancia para todos los involucrados en el sector portuario, desde las compañías navieras, autoridades portuarias, operadores hasta inversionistas. Esto debido a que, las decisión de aportar nuevas capacidades e inversiones en los terminales portuarios debe ser respaldada por una demanda potencial creciente por lo que los tomadores de decisiones deben contar con pronósticos de tráfico de proyecciones de demanda confiables como soporte para respaldar las decisiones relacionadas con la operatividad e inversión en infraestructura (Mohamed, 2016).

Para el caso peruano, las entidades encargadas de efectuar dichas proyecciones cuentan con pronósticos realizados en base a datos de frecuencia anual modelados principalmente por métodos de análisis de series temporales y/o mínimos cuadrados ordinarios los cuales relacionan las variables de tráfico de carga con variables exógenas representativas de la actividad económica del hinterland ${ }^{1}$.

Teniendo en cuenta la importancia de contar con proyecciones confiables sobre el movimiento de carga es que se plantea el objetivo de este trabajo de investigación, el cual es estimar y proyectar la demanda de tráfico de carga en el puerto del Callao para el periodo 2019 al 2023. Para ello,

\footnotetext{
1 Se define el hinterland como el área de influencia de un
} puerto. a diferencia de otros estudios, se aprovecha los datos de frecuencia mensual, así como el empleo de otras variables macroeconómicas relacionadas principalmente con la demanda para estimar y proyectar la demanda bajo la metodología de series de tiempo SARIMAX.

Al respecto, los resultados de dicha investigación podrían servir como insumo para mejorar las políticas públicas en cuanto a la determinación de tarifas portuarias en el puerto del Callao. Asimismo, para mejorar la realización de inversión en infraestructura portuaria por parte de los concesionarios y de los distintos actores involucrados en el sector portuario como las compañías navieras, autoridades portuarias, operadores y otros inversionistas.

En lo que respecta a la determinación de tarifas portuarias, actualmente, el mecanismo usado por el Organismo Supervisor de la Inversión en Infraestructura de Transporte de Uso Público (OSITRAN) para revisar las tarifas portuarias en el puerto del Callao se basa en las tarifas tope o RPI-X, donde buscan que las tarifas por los servicios prestados no excedan el precio tope estimado por el regulador durante un período regulatorio de 5 años. Para la aplicación de dicha metodología, el regulador viene empleando información histórica sobre inversiones, gastos e ingresos de la empresa regulada, así como otras variables de la economía. Sin embargo, la tarifa actualizada no toma en consideración las futuras inversiones y la demanda futura de carga que tendrán los concesionarios de las terminales portuarias del puerto del Callao. En ese sentido, la metodología SARIMAX, empleada en la presente investigación, podría proporcionar al regulador de mayores herramientas para evaluar metodologías prospectivas para las revisiones tarifarias, las cuales les permita incluir las proyecciones de demanda de tráfico en el puerto del Callao ${ }^{2}$.

La presente investigación, tiene la siguiente estructura, posterior a la introducción; la sección 2 , presenta una revisión de la literatura sobre investigaciones relacionadas con la proyección de demanda portuaria. En la sección 3 se presenta los datos y metodología a emplear. Finalmente en la sección 4 y 5 se muestran los resultados, la discusión de los mismos y las conclusiones de la investigación.

2 Por ejemplo, para el cálculo del Factor de Productividad de la empresa, el regulador podría evaluar la estimación del mismo bajo la metodología de Building Blocks. 


\section{REVISIÓN DE LA LITERATURA}

Los estudios relacionados al tema de proyecciones de demanda indican que, por lo general, los modelos ARIMA tienen una mayor inclinación a brindar mejores resultados ya que estos modelos, cuando son utilizados con datos de frecuencia trimestral o mensual, capturan de mejor manera el comportamiento de la demanda.

Dragan, Intihar y Kramberger (2014) realizaron un estudio en el que comparaban tres metodologías para proyectar el movimiento de carga en contenedores para los puertos NAPA (North Adriatic Ports Association). En dicho estudio se compararon modelos ARIMA, modelos de descomposición y modelos de suavizamiento exponencial Holt-Winters. Los resultados indicaron que los modelos ARIMA presentaban una mejor performance ante los otros modelos teniendo en cuenta como criterios principales de evaluación el RMSE, MAE y MAPE. Tomando como base dicho estudio, Dragan, Intihar y Kramberger (2017) realizaron otro estudio en el que se examinó el desempeño de los modelos ARIMAX exclusivamente para el puerto de Koper, Eslovenia, considerando variables exógenas representativas de la actividad económica del hinterland. Los resultados obtenidos, mostraron que al aplicar indicadores macroeconómicos en los modelos de proyección se puede lograr obtener mejores proyecciones a futuro del movimiento de carga.

Por su parte, Kotcharat (2016), realizó pronósticos basados en modelos SARIMAX tomando como variables internas índices de consumo privado, empleo, inversión privada y producción industrial; y como variables externas el precio del petróleo y el tipo de cambio. De acuerdo con sus resultados, el mejor modelo de proyección que obtuvo estuvo en función de tres variables exógenas (índice de inversión privada, empleo y el precio del petróleo), el cual fue comparado con otros modelos mediante los criterios RMSE, MAE, MAPE y el coeficiente de desigualdad de Theil.

La Tabla $\mathrm{N}^{\circ} 1$ presenta una revisión de los principales artículos que realizan proyecciones de demanda donde se señala, entre otras cosas, las variables empleadas y la metodología utilizada.

Tabla $N^{\circ}$ 1: Revisión de la literatura

\begin{tabular}{|c|c|c|c|c|c|c|}
\hline Autor & $\begin{array}{l}\text { Frecuencia } \\
\text { (periodo) }\end{array}$ & Ciudad/Región & $\begin{array}{l}\text { Variables } \\
\text { Endógena }\end{array}$ & Variables Exógenas & Objetivos & Metodología \\
\hline $\begin{array}{l}\text { Hui, Eddie; } \\
\text { Seabrooke, } \\
\text { William; Wong, } \\
\text { Gordon (2004). }\end{array}$ & $\begin{array}{l}1992 \mathrm{O}_{1}- \\
2001 \mathrm{O}_{4}\end{array}$ & $\begin{array}{l}\text { Puerto de Hong } \\
\text { Kong (China) }\end{array}$ & $\begin{array}{l}\text { Cargo } \\
\text { Throughput } \\
\text { (millones de } \\
\text { toneladas) }\end{array}$ & $\begin{array}{l}\text {-Valor comercial total de China. } \\
\text {-Valor comercial total de EE.UU. } \\
\text {-Número de amarres de terminales de } \\
\text { contenedores. } \\
\text {-Cargo Throughput de los puertos de } \\
\text { Shenzhen. }\end{array}$ & $\begin{array}{l}\text { Proveer mejoras en las proyecciones de } \\
\text { movimiento de carga realizadas por las } \\
\text { autoridad portuarias regionales con el fin } \\
\text { de mejorar el panorama de inversiones en } \\
\text { infraestructura. }\end{array}$ & $\begin{array}{l}\text {-Modelo de Corrección de Errores } \\
(E C M)\end{array}$ \\
\hline $\begin{array}{l}\text { Wen-Yi Peng, } \\
\text { Ching-Wu Chu } \\
\text { (2009) }\end{array}$ & $\begin{array}{l}\text { January } \\
\text { 2003- } \\
\text { December } \\
2006\end{array}$ & $\begin{array}{l}\text { Los Tres } \\
\text { principales } \\
\text { puertos de } \\
\text { Taiwán }\end{array}$ & $\begin{array}{l}\text {-Volúmenes de } \\
\text { rendimiento de } \\
\text { contenedores } \\
\text { para los puertos } \\
\text { de Keelung, } \\
\text { Taichungy } \\
\text { Kaohsiung (TEU's) }\end{array}$ & $\begin{array}{l}\text {-Factor Tendencial. } \\
\text {-Factor cíclico. } \\
\text {-Factor estacional. } \\
\text {-Factor irregular. }\end{array}$ & $\begin{array}{l}\text { Encontrar un modelo que pueda } \\
\text { proporcionar la mayor predicción precisa } \\
\text { del rendimiento de un contenedor. } \\
\text { Comparando seis modelos univariados de } \\
\text { predicción. }\end{array}$ & $\begin{array}{l}\text { - Modelo clásico de } \\
\text { descomposición. } \\
\text {-Modelo de Regresión } \\
\text { Trigonométrica. } \\
\text {-Modelo de regresión con variables } \\
\text { ficticias estacionales. } \\
\text {-El modelo gris. } \\
\text {-Modelo Gris Híbrido. } \\
\text {-Modelo SARIMA. } \\
\end{array}$ \\
\hline $\begin{array}{l}\text { Peter M. Schulze, } \\
\text { Alexander Prinz } \\
\text { (2010). }\end{array}$ & $\begin{array}{l}19890_{1}- \\
20060_{4}\end{array}$ & Alemania & $\begin{array}{l}\text { Twenty-foot } \\
\text { Equivalent Units } \\
\text { (TEU's) }\end{array}$ & $\begin{array}{l}\text {-Constante. } \\
\text {-Seasonal Dummy. }\end{array}$ & $\begin{array}{l}\text { El propósito de la investigación es } \\
\text { encontrar el mejor modelo, entre los dos } \\
\text { seleccionados para predecir de manera } \\
\text { más eficiente la previsibilidad. } \\
\text { Comparando los modelos a través del } \\
\text { RMSE y la U de Theil. }\end{array}$ & $\begin{array}{l}\text {-SARIMA. } \\
\text {-The Holt-Winters Exponential } \\
\text { Smoothing Approach. }\end{array}$ \\
\hline $\begin{array}{l}\text { Shih-Huang Chen, } \\
\text { Jun-Nan Chen } \\
\text { (2010). }\end{array}$ & $\begin{array}{l}1978 \\
\text { January to } \\
2006 \\
\text { Diciembre }\end{array}$ & Taiwan & $\begin{array}{l}\text { Twenty-foot } \\
\text { Equivalent Units } \\
\text { (TEU'S) }\end{array}$ & - & $\begin{array}{l}\text { El presente estudio pretende crear un } \\
\text { modelo óptimo para el rendimiento por } \\
\text { volumen del contenedor en los puertos } \\
\text { usando distintas metodologías, } \\
\text { comparándolos a través del MAPE. }\end{array}$ & $\begin{array}{l}\text { - Programación Genética. -Enfoque } \\
\text { de descomposición (X-11).- } \\
\text { SARIMA. }\end{array}$ \\
\hline $\begin{array}{l}\text { Veerachai } \\
\text { Gosasang, } \\
\text { Watcharavee } \\
\text { Chandraprakaikul } \\
\text {, Supaporn } \\
\text { Kiattisin (2011). }\end{array}$ & $1999-2010$ & $\begin{array}{l}\text { Tailandia, puerto } \\
\text { de Bangkok. }\end{array}$ & $\begin{array}{l}\text {-Número de } \\
\text { contenedores en } \\
\text { el puerto de } \\
\text { Bangkok. } \\
\text {-Importaciones. } \\
\text {-Exportaciones. }\end{array}$ & $\begin{array}{l}\text {-Población. } \\
\text {-PBI nacional. } \\
\text {-PBI mundial. } \\
\text {-Tipo de Cambio. } \\
\text {-Inflación. } \\
\text {-Tasa de Interés. } \\
\text {-Precio del Petróleo. }\end{array}$ & $\begin{array}{l}\text { Explorar un Perceptron Multicapa (MLP) } \\
\text { y una regresión lineal para para predecir } \\
\text { el rendimiento futuro de los } \\
\text { contenedores en el puerto de Bangkok. }\end{array}$ & $\begin{array}{l}\text {-Multilayer Perceptron. } \\
\text {-Modelo de regresión Lineal. }\end{array}$ \\
\hline
\end{tabular}




\begin{tabular}{|c|c|c|c|c|c|c|}
\hline $\begin{array}{l}\text { Dragan, Dejan; } \\
\text { Intihar, Marko; } \\
\text { Kramberger, } \\
\text { Tomaz (2014). }\end{array}$ & $\begin{array}{l}2002 \mathrm{O}_{1} \\
2012 \mathrm{O}_{2}\end{array}$ & $\begin{array}{l}\text { North Adriatic } \\
\text { Ports Association } \\
\text { (NAPA); Puerto } \\
\text { de Koper } \\
\text { (Eslovenia) - } \\
\text { Puertos de } \\
\text { Venecia, Trieste } \\
\text { \& Ravenna (Italia) } \\
\end{array}$ & $\begin{array}{l}\text { Twenty-foot } \\
\text { Equivalent Units } \\
\text { (TEU's) }\end{array}$ & - & $\begin{array}{l}\text { Comparar } 3 \text { métodos para proyectar } \\
\text { movimiento de carga en contenedores } \\
\text { con la finalidad de optimizar operaciones } \\
\text { y uso de facilidades portuarias. }\end{array}$ & $\begin{array}{l}\text {-Modelos de Descomposición. } \\
\text {-Modelos ARIMA. } \\
\text {-Modelos de Suavizamiento } \\
\text { Exponencial de Holt-Winters. }\end{array}$ \\
\hline $\begin{array}{l}\text { Mohamed, } \\
\text { Yasmine R. } \\
\text { (2016). }\end{array}$ & $\begin{array}{l}1995 \mathrm{M}_{1}- \\
2015 \mathrm{M}_{3}\end{array}$ & $\begin{array}{l}\text { Puerto de } \\
\text { Antwerp (Bélgica) }\end{array}$ & $\begin{array}{l}\text { Twenty-foot } \\
\text { Equivalent Units } \\
\text { (TEU's) }\end{array}$ & $\begin{array}{l}\text { Indicadores de Confianza: } \\
\text {-Composite Index of Leading Indicators. } \\
\text {-Economic Sentiment Indicator. } \\
\text {-Industrial Confidence Indicator. } \\
\text { Indicadores de Comercio e Industria: } \\
\text {-Indice de Producción Industrial. } \\
\text {-Indice de Volumen de Exportaciones } \\
\text { Totales. } \\
\text {-Indice de Volumen Importaciones } \\
\text { Totales. }\end{array}$ & $\begin{array}{l}\text { Proveer un instrumento cuantitativo de } \\
\text { apoyo para decisiones operativas de } \\
\text { corto plazo y decisiones de inversión de } \\
\text { largo plazo. }\end{array}$ & $\begin{array}{l}\text {-Modelos ARIMA/ARIMAX. } \\
\text {-Modelos de Cointegración. } \\
\text {-Modelos Autorregresivos de } \\
\text { Rezagos Distribuidos. }\end{array}$ \\
\hline $\begin{array}{l}\text { Dragan, Dejan; } \\
\text { Intihar, Marko; } \\
\text { Kramberger, } \\
\text { Tomaz (2017). }\end{array}$ & $\begin{array}{l}2002 \mathrm{O}_{1} \\
20160_{4}\end{array}$ & $\begin{array}{l}\text { Puerto de Koper } \\
\text { (Eslovenia) }\end{array}$ & $\begin{array}{l}\text { Twenty-foot } \\
\text { Equivalent Units } \\
\text { (kilotons) }\end{array}$ & $\begin{array}{l}\text {-PBI per cápita } \\
\text {-Paridad de Poder Adquisitivo } \\
\text {-Exportaciones (miles de millones USD) } \\
\text {-Importaciones (miles de millones USD) } \\
\text {-Desempleo(\%) }\end{array}$ & $\begin{array}{l}\text { Examinar el impacto de la integración de } \\
\text { indicadores macroeconómicos en la } \\
\text { precisión del modelo de pronóstico de } \\
\text { series de tiempo de movimiento de } \\
\text { contenedores. }\end{array}$ & $\begin{array}{l}\text {-Modelos ARIMAX. } \\
\text {-Análisis de Factores Dinámicos. }\end{array}$ \\
\hline
\end{tabular}

Fuente y Elaboración: Propia

\section{DATOS Y METODOLOGÍA}

La población está definida como la base de datos de la Autoridad Portuaria Nacional (APN) sobre el movimiento de carga para el Sistema Portuario Nacional, la cual cuenta con información de movimiento de carga para puertos nacionales (de uso público y privado) desagregada por tipo de operación y tipo de carga en periodicidad mensual. Dicha información cuenta con cifras de movimiento de carga a partir del año 2007. Para datos previos al 2007 se recurrió a los boletines estadísticos de la Empresa Nacional de Puertos (ENAPU), los cuales presentaban datos históricos desde el año 1999 en frecuencia mensual ${ }^{3}$.

La muestra obtenida está compuesta por las diversas series de movimiento de carga para el Puerto del Callao en sus respectivas unidades: toneladas métricas (TM) o twenty-foot equivalent units (TEU's). Es decir, el movimiento de carga del Terminal Norte Multipropósito (APM
Terminals Callao - APMTC), el Terminal de Contenedores Muelle Sur (Dubái Ports World Callao - DPWC) y el Terminal de Embarque de Concentrado de Minerales (Transportadora Callao - TC). Cabe mencionar que, para el caso del Terminal de Embarque de Concentrado de Minerales y el Terminal de Contenedores Muelle Sur, el tamaño muestral refleja su nivel de actividad desde el inicio de sus operaciones hasta la actualidad.

Las variables exógenas empleadas en este artículo provienen del Banco Central de Reserva del Perú. El grupo de variables exógenas está compuesto básicamente de indicadores comerciales, índices de confianza y precios de commodities (cobre). Para la elaboración de los modelos de proyección se emplearon variables exógenas representativas de la actividad económica, las cuales aportan a la obtención de mejores resultados de proyección. Las variables empleadas en este estudio son las siguientes:

3 A pesar de contar con disponibilidad de datos desde 1999 se optó por considerar información histórica a partir del año 2003 debido cambios en el proceso de consolidación de datos. 
TABLA N²: LISTADO DE VARIABLES ENDÓGENAS Y EXÓGENAS

\begin{tabular}{|c|c|c|}
\hline \multirow[t]{3}{*}{$\begin{array}{l}\text { Variables } \\
\text { Endógenas }\end{array}$} & $\begin{array}{c}\text { Terminal Norte Multipropósito } \\
\text { (APMTC) }\end{array}$ & $\begin{array}{c}\text { Carga Fraccionada (TM) • } \\
\text { Granel Sólido (TM) • } \\
\text { Granel Líquido (TM) • } \\
\text { Contenedores (TEU) • } \\
\text { Carga Rodante }(\mathrm{TM}) \bullet\end{array}$ \\
\hline & $\begin{array}{c}\text { Terminal de Contenedores Muelle } \\
\text { Sur (DPWC) }\end{array}$ & Contenedores (TEU's) • \\
\hline & $\begin{array}{c}\text { Terminal de Embarque de } \\
\text { Concentrado de Minerales (TC) }\end{array}$ & Granel Sólido (TM) • \\
\hline \multirow[t]{3}{*}{ Variables Exógenas } & Indicadores Comerciales & $\begin{array}{l}\text { Importación de Bienes de Capital (millones de US\$) } \\
\text { Importación de Bienes de Consumo (millones de US\$) } \\
\text { Exportación de Productos Tradicionales (millones de US\$) } \\
\text { Índice de Apertura Comercial (\%) }\end{array}$ \\
\hline & Índices de Confianza & Índice de Expectativas de la Economía a 3 meses (\%) \\
\hline & Precios de Commodities & Copper Price (cUS\$ por libra) \\
\hline
\end{tabular}

Fuente y Elaboración: Propia

La siguiente tabla muestra la estadística descriptiva de las variables utilizadas en este estudio:

Tabla $N^{\circ} 3$ : Estadistica Descriptiva de las variables empleadas

\begin{tabular}{|c|c|c|c|c|c|c|c|}
\hline \multirow{6}{*}{$\begin{array}{c}\text { Terminal Norte } \\
\text { Multipropósito } \\
\text { (APMTC) }\end{array}$} & $\begin{array}{c}\text { Variable } \\
\text { (unidades) }\end{array}$ & Periodo & $\begin{array}{c}N^{\circ} \text { de } \\
\text { Observaciones }\end{array}$ & Promedio & Mediana & Máximo & Mínimo \\
\hline & $\begin{array}{c}\text { Carga } \\
\text { Fraccionada } \\
(\mathrm{TM})\end{array}$ & $2003 Q 1-2018 Q 4$ & 64 & $340,288.40$ & $371,527.00$ & $657,184.00$ & $78,926.00$ \\
\hline & $\begin{array}{l}\text { Granel Sólido } \\
\text { (TM) }\end{array}$ & $2003 Q 1-2018 Q 4$ & 64 & $1,349,933.00$ & $1,365,234.00$ & $1,810,838.00$ & $896,752.00$ \\
\hline & $\begin{array}{c}\text { Granel Líquido } \\
\text { (TM) }\end{array}$ & $2003 Q 1-2018 Q 4$ & 64 & $254,967.10$ & $597,073.50$ & $875,086.00$ & $225,157.00$ \\
\hline & $\begin{array}{c}\text { Twenty-foot } \\
\text { Equivalent } \\
\text { Units (TEU's) }\end{array}$ & 2003M1-2018M12 & 192 & $65,463.03$ & $66,923.50$ & $112,364.00$ & $21,617.00$ \\
\hline & $\begin{array}{c}\text { Carga Rodante } \\
\text { (TM) }\end{array}$ & $2003 Q 1-2018 Q 4$ & 64 & $60,037.20$ & $69,551.00$ & $123,847.00$ & $4,649.00$ \\
\hline $\begin{array}{l}\text { Terminal de } \\
\text { Contenedores } \\
\text { Muelle Sur } \\
\text { (DPWC) }\end{array}$ & $\begin{array}{c}\text { Twenty-foot } \\
\text { Equivalent } \\
\text { Units (TEU's) }\end{array}$ & $2010 M 06-2018 M 12$ & 103 & $101,268.70$ & $103,737.00$ & $126,506.00$ & $25,304.00$ \\
\hline $\begin{array}{c}\text { Terminal de } \\
\text { Embarque de } \\
\text { Concentrado de } \\
\text { Minerales (TC) }\end{array}$ & $\begin{array}{l}\text { Granel Sólido } \\
\text { (TM) }\end{array}$ & $2014 M 06-2018 M 12$ & 55 & $252,434.70$ & $246,351.90$ & $401,851.80$ & $154,526.60$ \\
\hline
\end{tabular}




\begin{tabular}{|c|c|c|c|c|c|c|c|}
\hline \multirow{6}{*}{$\begin{array}{l}\text { Variables } \\
\text { Exógenas }\end{array}$} & $\begin{array}{c}\text { Importación } \\
\text { de Bienes } \\
\text { de Capital } \\
\text { (millones US\$) }\end{array}$ & $2003 Q 1-2018 Q 4$ & 64 & $2,193.35$ & $2,575.83$ & $3,562.61$ & 465.93 \\
\hline & $\begin{array}{c}\text { Importación } \\
\text { de Bienes de } \\
\text { Consumo } \\
\text { (millones US\$) }\end{array}$ & $2003 Q 1-2018 Q 4$ & 64 & $1,483.14$ & $1,533.06$ & $2,498.39$ & 413.09 \\
\hline & $\begin{array}{c}\text { Exportación } \\
\text { de Productos } \\
\text { Tradicionales } \\
\text { (millones US\$) }\end{array}$ & 2003M1-2018M12 & 192 & $2,031.63$ & $2,145.87$ & $3,613.35$ & 429.15 \\
\hline & $\begin{array}{c}\text { Índice de } \\
\text { Expectativas de } \\
\text { la Economía a } 3 \\
\text { Meses (\%) }\end{array}$ & $2003 Q 1-2018 Q 4$ & 64 & 58.98 & 58.76 & 73.33 & 35.33 \\
\hline & $\begin{array}{c}\text { Índice de } \\
\text { Apertura } \\
\text { Comercial (\%) }\end{array}$ & $2000 Q 1-2018 Q 4$ & 76 & 0.46 & 0.47 & 0.61 & 0.32 \\
\hline & $\begin{array}{l}\text { Copper Price } \\
\text { (eUS\$ por } \\
\text { libra) }\end{array}$ & 2014M06-2018M12 & 55 & 267.41 & 269.50 & 322.25 & 202.43 \\
\hline
\end{tabular}

Fuente y Elaboración: Propia

Con estas variables se realiza un análisis cuantitativo, prospectivo de las diversas series de movimiento de carga para el puerto del Callao con el objetivo de proveer proyecciones para el establecimiento de tarifas.

En el presente trabajo de investigación se pretende emplear distintas metodologías basadas en la familia de modelos ARIMA. La implementación la metodología ARIMA fue introducida por Box y Jenkins (1976), la cual tiene como objetivo principal determinar un modelo univariado apropiado con el propósito de estimar y proyectar una serie de tiempo. Esta metodología consiste en tres pasos principales:

\section{i. Identificación}

Mediante una inspección visual se analiza la gráfica de la serie, las funciones de autocorrelación (ACF) y autocorrelación parcial (PACF). Al hacer esto se puede identificar valores extremos, datos faltantes y quiebres estructurales en la data. Variables no-estacionarias pueden exhibir tendencias pronunciadas o pueden presentar un comportamiento sin media o varianza aparente a largo plazo. El procedimiento estándar sugiere diferenciar las series que aparenten no ser estacionarias, para ello se aplican transformaciones a la serie con la finalidad de llegar a la condición de estacionariedad. Esto se comprueba mediante pruebas de raíz unitaria, las cuales determinan si la serie resulta estacionaria antes y después de la transformación.

\section{ii. Estimación}

En esta etapa se ponen a prueba los modelos tentativos y se examinan los parámetros estimados. El objetivo consiste en seleccionar un modelo estacionario y parsimonioso que tenga un buen ajuste. Para ello se emplean criterios de selección de modelos tales como el criterio de información de Akaike (AIC), criterio de información de Schwarz (SC) y/o el Final Prediction Error (FPE) los cuales permiten hacen comparaciones entre distintas especificaciones y de esta manera seleccionar el mejor modelo.

\section{iii. Diagnóstico}

En esta última etapa se comprueba la idoneidad del modelo. Se requiere que los residuos del modelo estimado sigan (o se asemejen a) un proceso de ruido blanco, para ello se examinan los residuos con la finalidad de determinar si de hecho exhibe el comportamiento esperado. Con la ayuda de las funciones de autocorrelación y autocorrelación parcial se determina que no existan autocorrelaciones significativas. Adicionalmente a ello se pueden aplicar pruebas de Ljung-Box lo cual demuestra que los residuos se pueden considerar provenientes de un proceso de ruido blanco (Enders, 2014). 
Tabla $\mathbf{N}^{\circ}$ 4: Resumen de procedimiento metodológico Box-Jenkins

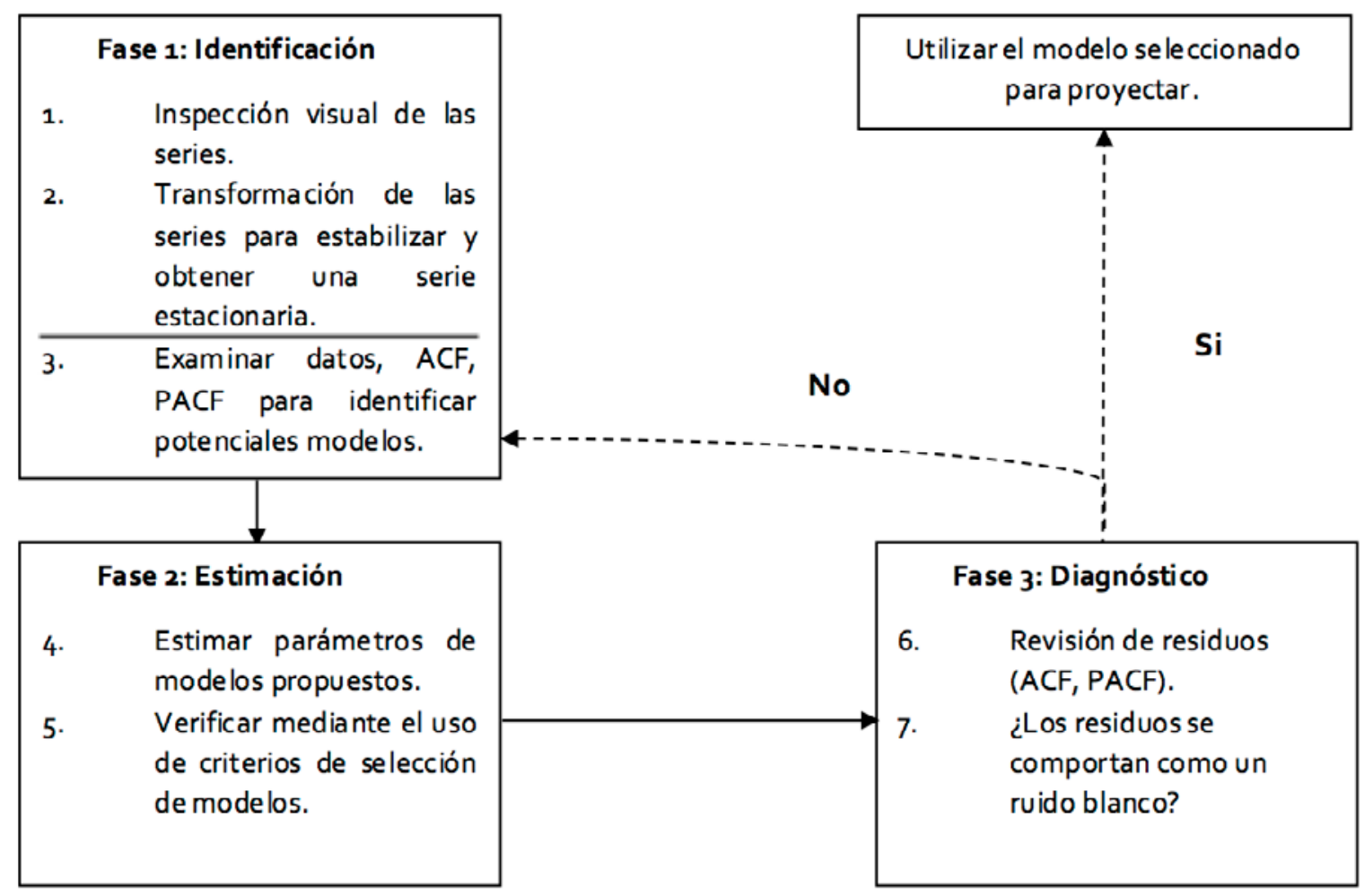

Fuente y Elaboración: Propia

Los modelos ARIMAX, al igual que los modelos ARIMA, están compuestos por componentes auto regresivos (AR), medias móviles (MA) y el componente de integración. La principal diferencia consiste en la inclusión de inputs exógenos en el modelo. De esta manera, el modelo ARIMAX consiste principalmente en encontrar una variable exógena representativa de la serie de movimiento de contenedores. Teniendo en cuenta que existe una relación entre la actividad económica y el movimiento de contenedores (Mohamed, 2016).

Por otro lado, los modelos SARIMA, los cuales forman parte de la familia ARIMA, se utilizan ampliamente con datos de series temporales estacionales lo cual implica que presenta patrones de comportamientos similares a través del tiempo. Por ejemplo, en datos mensuales, los valores tienden a ser altos en algunos meses en particular y bajos otros (Kotcharat, 2016).

Teniendo en cuenta que los modelos previamente mencionados deben cumplir los supuestos básicos de MCO y considerando que podrían presentarse series volátiles es que, además de los modelos propuestos, se considera la posible inclusión de modelos GARCH con la finalidad de eliminar problemas de heterocedasticidad. Los modelos GARCH son una generalización de los modelos $\mathrm{ARCH}$, y son los más usados para modelar la volatilidad. Esta generalización fue realizada por Bollerslev (1986) y como pertenece a la familia de los modelos ARCH son también de especial uso en lo que respecta a los procesos de tiempo discretos; es decir, también sirven para portafolios, y/o instrumentos financieros.

Por lo tanto, para el presente artículo se procede de la siguiente manera:

Primero, en la etapa de identificación, se analiza la estacionariedad de las series, para ello se realizan pruebas de raíz unitaria de series temporales tales como: Augmented DickeyFuller (ADF) y Phillips-Perron (PP). Si los resultados indican que las series presentan raíz unitaria se procederá a aplicar transformaciones a las variables (primeras diferencias, logaritmos, variaciones porcentuales). Una vez que se disponga de variables estacionarias se procede a realizar una revisión visual de las funciones 
de autocorrelación y autocorrelación parcial con la finalidad de proponer e identificar posibles modelos explicativos de las series de movimiento de carga.

En la etapa de estimación se seleccionan los modelos que presentan mejores indicadores sobre criterios de selección tales como: Akaike Information Criterion y Schwarz Information Criterion y se revisa la significancia de los parámetros estimados. Posteriormente, se procede a incorporar los componentes estacionales SAR y SMA y las variables exógenas necesarias representativas de la actividad económica explicativas del movimiento de carga de los terminales portuarios para mejorar los modelos de proyección realizados. Cabe mencionar que para la fase de estimación se realiza proyecciones dentro y fuera de muestra con la finalidad de contar con un intervalo de prueba al que serán sometidos los modelos seleccionados. Se propone tomar el $80 \%$ de la data como submuestra para el proceso de estimación y tomar el $20 \%$ restante como intervalo de prueba.

Seguidamente, para realizar la etapa de diagnóstico, se procede a analizar los residuos de regresión los cuales deben exhibir un comportamiento similar al de un proceso de ruido blanco. Comprobadas estas condiciones se procede a realizar proyecciones a periodos futuros. Adicionalmente se toma en consideración indicadores de evaluación de proyecciones tales como el error cuadrático medio, error porcentual medio absoluto, coeficiente de desigualdad de Theil.

Para la realización de las estimaciones y proyecciones se emplea el software econométrico EViews 10.0.

\section{RESULTADOS}

En primer lugar, la Tabla $\mathrm{N}^{\circ} 5$ presentan las pruebas de raíz unitaria por tipo de carga para cada terminal del Puerto del Callao: (APMTC, DPWC y TC)

Tal como se observa, según la hipótesis nula de las pruebas de Dickey-Fuller y Phillips-Perron, que es que las series presentan raíz unitaria, se observa que todas las series presentan raíz unitaria y son estacionarias en primeras diferencias, a excepción de APMTC - graneles sólidos, DPWC - contenedores (TEU's), TC - graneles sólidos, índice de expectativas de la economía a 3 meses e índice de Apertura Comercial.

Tabla $N^{\circ}$ 5: Pruebas de Raiz Unitaria

\begin{tabular}{|c|c|c|c|}
\hline Variable & Método & Niveles & Diferencias \\
\hline \multicolumn{4}{|c|}{ Endógenas } \\
\hline \multirow{2}{*}{ APMTC - Carga Fraccionada (TM) • } & Augmented Dickey-Fuller (ADF) & -0.790 & $-8.325 * * *$ \\
\hline & Phillips-Perron & -1.052 & $-11.960 * * *$ \\
\hline \multirow{2}{*}{ APMTC - Graneles Sólidos (TM) • } & Augmented Dickey-Fuller (ADF) & $-5.491 * * *$ & $-7.977 * * *$ \\
\hline & Phillips-Perron & $-5.489 * * *$ & $-31.812 * * *$ \\
\hline \multirow{2}{*}{ APMTC - Graneles Líquidos (TM)• } & Augmented Dickey-Fuller (ADF) & -2.192 & $-10.447 * * *$ \\
\hline & Phillips-Perron & -1.822 & $-14.610 * * *$ \\
\hline \multirow{2}{*}{ APMTC - Contenedores (TEU) } & Augmented Dickey-Fuller (ADF) & -1.979 & $-19.220 * * *$ \\
\hline & Phillips-Perron & -2.232 & $-19.220 * * *$ \\
\hline \multirow{2}{*}{ APMTC - Carga Rodante (TM) • } & Augmented Dickey-Fuller (ADF) & -1.564 & $-6.643 * * *$ \\
\hline & Phillips-Perron & -1.473 & $-6.746^{* * *}$ \\
\hline \multirow{2}{*}{ DPWC - Contenedores (TEU's) • } & Augmented Dickey-Fuller (ADF) & $-3.980 * * *$ & $-9.774 * * *$ \\
\hline & Phillips-Perron & $-3.808 * * *$ & $-14.616^{* * *}$ \\
\hline \multirow{2}{*}{ TC - Graneles Sólidos (TM) • } & Augmented Dickey-Fuller (ADF) & $-8.487 * * *$ & $-6.833 * * *$ \\
\hline & Phillips-Perron & $-8.538 * * *$ & $-30.338 * * *$ \\
\hline \multicolumn{4}{|c|}{ Exógenas } \\
\hline \multirow{2}{*}{$\begin{array}{l}\text { Importaciones de Bienes de Capital } \\
\text { (millones de US\$) }\end{array}$} & Augmented Dickey-Fuller (ADF) & -1.466 & $-6.329 * * *$ \\
\hline & Phillips-Perron & -1.467 & $-6.185^{* * *}$ \\
\hline
\end{tabular}




\begin{tabular}{|c|c|c|c|}
\hline \multirow{2}{*}{$\begin{array}{l}\text { Importaciones de Consumo (millones de } \\
\text { US\$) }\end{array}$} & Augmented Dickey-Fuller (ADF) & -1.168 & $-2.868^{*}$ \\
\hline & Phillips-Perron & -0.702 & $-9.530 * * *$ \\
\hline \multirow{2}{*}{$\begin{array}{l}\text { Exportaciones de Productos Tradicionales } \\
\text { (millones de US\$) }\end{array}$} & Augmented Dickey-Fuller (ADF) & -1.809 & $-7.248 * * *$ \\
\hline & Phillips-Perron & -1.792 & $-7.706^{* * *}$ \\
\hline \multirow{2}{*}{$\begin{array}{l}\text { Índice de Expectativas de la Economía a } 3 \\
\text { meses (\%) }\end{array}$} & Augmented Dickey-Fuller (ADF) & $-4.393 * * *$ & $-6.825^{* * *}$ \\
\hline & Phillips-Perron & $-3.164 * *$ & $-6.596^{* * *}$ \\
\hline \multirow{2}{*}{ Índice de Apertura Comercial (\%) } & Augmented Dickey-Fuller (ADF) & $-3.061^{* *}$ & $-2.995^{* *}$ \\
\hline & Phillips-Perron & $-2.965^{* *}$ & $-11.795^{* * *}$ \\
\hline \multirow{2}{*}{ Copper Price (cUS\$ por libra) } & Augmented Dickey-Fuller (ADF) & -1.428 & $-5.483 * * *$ \\
\hline & Phillips-Perron & -1.544 & $-5.458 * * *$ \\
\hline
\end{tabular}

$* * *$ Significativo al $1 \%, * *$ Significativo al $5 \%, *$ Significativo al $10 \%$

Fuente y Elaboración: Propia

La Tabla $\mathrm{N}^{\circ} 6$ muestra el resultado del mejor modelo obtenido para la carga fraccionada de APMTC, según lo detallado en la sección metodológica. El modelo incluye los componentes $\mathrm{AR}(1), \mathrm{AR}(2), \mathrm{SAR}(3), \mathrm{MA}(2)$. Asimismo, contempla la inclusión de la variable importación de bienes de capital y de dos variables dummy para los periodos 2010 y 2017-2018. Esto último debido a que durante los periodos previamente mencionados la serie de carga presenta valores que se desvían de la tendencia central o esta es afectada por periodos de crisis/recesión económica.

Tabla Nº: APMTC-Estimación del Modelo de Carga Fraccionada

\begin{tabular}{cc|cc}
\multicolumn{4}{c}{ Terminal Portuario: APMTC } \\
Método: ARMA Maximum Likelihood (BFGS) \\
Variable Dependiente: DLOG(FRACCIONADA)
\end{tabular}

$* * *$ Significativo al $1 \%, * *$ Significativo al $5 \%$, *Significativo al $10 \%$ 
El Gráfico $\mathrm{N}^{\circ} 1$, presenta la proyección de carga fraccionada para el periodo 2019-2023.

\section{Gráfico $N^{\circ}$ 1: APMTC-Proyección de Carga Fraccionada (TM)}

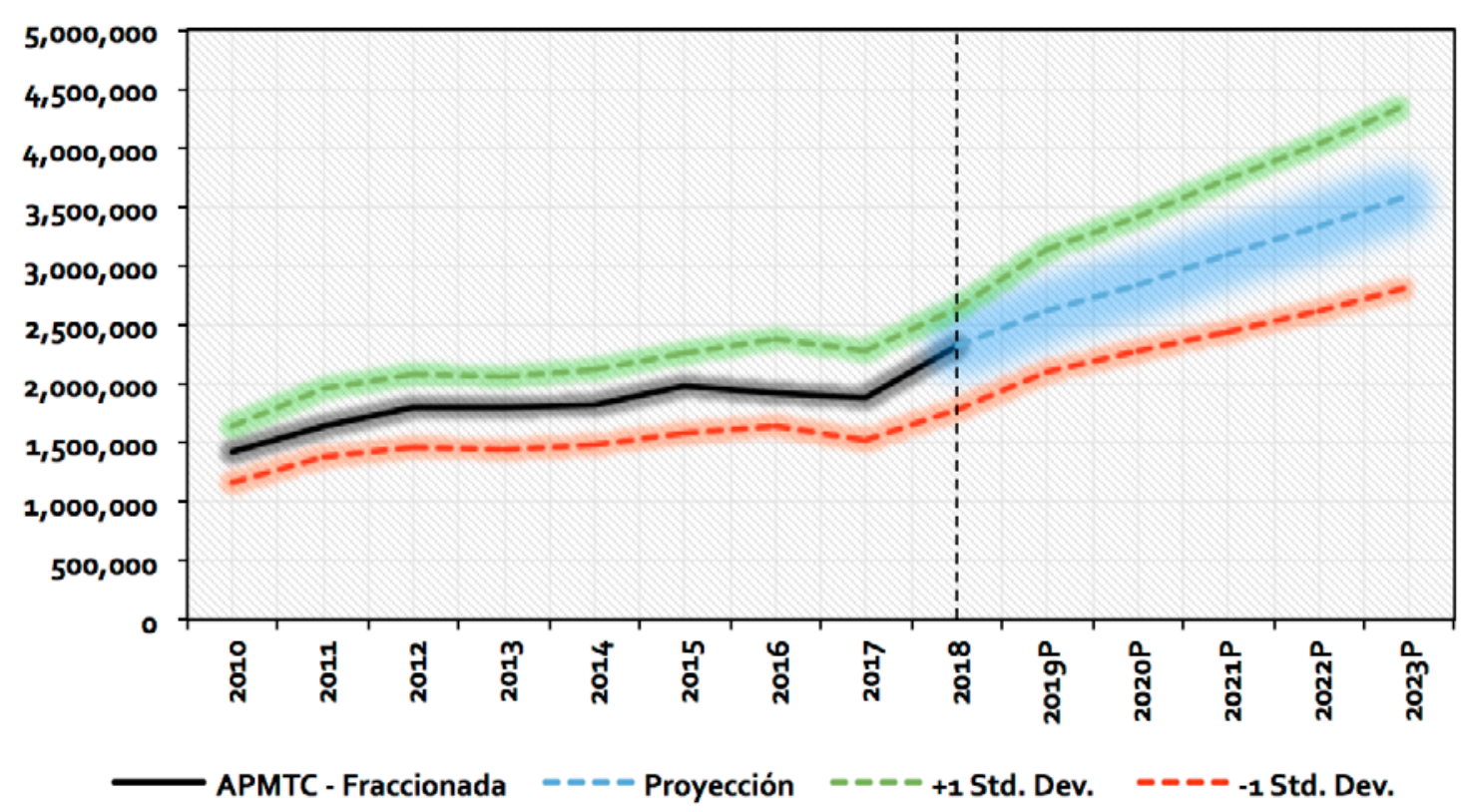

Fuente y Elaboración: Propia

La Tabla $\mathrm{N}^{\circ} 7$ presenta el resultado del mejor modelo obtenido para la carga de granel sólido de APMTC y TC, según lo detallado en la sección metodológica. La especificación para APMTC incluye los componentes AR(1), AR(2), $\operatorname{AR}(3), \operatorname{AR}(6)$ y SAR(4). Asimismo, contempla la inclusión de dos variables dummy para los periodos de 2010 y 2014 a 2015 . La primera variable para hacer correcciones producto de la crisis internacional y la segunda variable por la apertura del Terminal de Embarque de Concentrado de Minerales (TC). Respecto a las variables explicativas relevantes se consideró a la exportación de productos tradicionales, la importación de bienes de consumo y la importación de bienes de capital.

Por su parte para TC, teniendo en consideración que las operaciones de TC son exclusivamente de embarque (exportación) el mejor modelo obtenido considera la exportación de productos tradicionales y el precio del cobre. Asimismo, incluye dos variables dummy para algunos periodos de los años 2015 y 2016 donde se registraron valores extremos. 
Tabla N²: APMTC y TC-Estimación del Modelo de Carga de Graneles Sólidos

\begin{tabular}{|c|c|c|c|c|c|c|c|}
\hline \multicolumn{4}{|c|}{$\begin{array}{c}\text { Terminal Portuario: APMTC } \\
\text { Método: ARMA Maximum Likelihood (BFGS) } \\
\text { Variable Dependiente: LOG(GSOL) }\end{array}$} & \multicolumn{4}{|c|}{$\begin{array}{c}\text { Terminal Portuario: TC } \\
\text { Método: ARMA Maximum Likelihood (BFGS) } \\
\text { Variable Dependiente: LOG(GSOL) }\end{array}$} \\
\hline Variable & $\begin{array}{l}\text { Coeficiente } \\
\text { / Desv. Est. }\end{array}$ & Variable & $\begin{array}{l}\text { Coeficiente / } \\
\text { Desv. Est. }\end{array}$ & Variable & & Coeficient & / Desv. Est. \\
\hline$C$ & $\begin{array}{c}0.003 \\
(0.005)\end{array}$ & $A R(1)$ & $\begin{array}{c}-1.169^{* * *} \\
(0.161)\end{array}$ & $\mathrm{C}$ & & $\begin{array}{r}12.4 \\
(0 .\end{array}$ & $\begin{array}{l}8 * * * \\
19)\end{array}$ \\
\hline $\begin{array}{l}\text { DLOG(EXP_- } \\
\text { PROD_TRAD) }\end{array}$ & $\begin{array}{c}0.320^{* * *} \\
(0.053)\end{array}$ & $\operatorname{AR}(2)$ & $\begin{array}{c}-0.879 * * * \\
(0.209)\end{array}$ & $\begin{array}{r}\text { DLOG(EXP_Pl } \\
\text { TRAD) }\end{array}$ & $\mathrm{ROD}_{-}$ & $\begin{array}{r}0.62 \\
(0 .\end{array}$ & $\begin{array}{l}* * * \\
54)\end{array}$ \\
\hline $\begin{array}{l}\text { DLOG(IMP } \\
\text { CONSUMO) }\end{array}$ & $\begin{array}{c}0.649 * * * \\
(0.153)\end{array}$ & $\operatorname{AR}(3)$ & $\begin{array}{c}-0.618^{* * *} \\
(0.146)\end{array}$ & $\begin{array}{r}\text { DLOG(COPP } \\
\text { PRICE_F }\end{array}$ & ER_- & $\begin{array}{r}-2.2 \\
(0 .\end{array}$ & $\begin{array}{l}5 * * * \\
07)\end{array}$ \\
\hline $\begin{array}{l}\text { DLOG(IMP } \\
\text { CAPITAL) }\end{array}$ & $\begin{array}{c}-0.490 * * * \\
(0.092)\end{array}$ & $\operatorname{AR}(6)$ & $\begin{array}{l}-0.152 * \\
(0.083)\end{array}$ & DUMMY1 & & $\begin{array}{r}0.32 \\
(0 .\end{array}$ & $\begin{array}{l}* * * \\
99)\end{array}$ \\
\hline DUMMY3 & $\begin{array}{c}-0.283 * * * \\
(0.053)\end{array}$ & $\operatorname{SAR}(4)$ & $\begin{array}{c}-0.573 * * \\
(0.168)\end{array}$ & DUMMY 1 & & $\begin{array}{r}0.45 \\
(0 .\end{array}$ & $\begin{array}{l}* * * * \\
94)\end{array}$ \\
\hline DUMMY4 & $\begin{array}{c}-0.048 * * * \\
(0.011)\end{array}$ & SIGMASQ & $\begin{array}{c}0.005 * * * \\
(0.001)\end{array}$ & & & & \\
\hline $\begin{array}{l}\text { Observaciones } \\
\text { Incluidas }\end{array}$ & 63 & $\begin{array}{l}\text { Periodos } \\
\text { Proyectados }\end{array}$ & 20 & $\begin{array}{l}\text { Observaciones } \\
\text { Incluidas }\end{array}$ & 54 & $\begin{array}{l}\text { Periodos } \\
\text { Proyectados }\end{array}$ & 20 \\
\hline $\mathrm{R}^{2}$ & 0.7957 & RMSE & $107,216.3000$ & $\mathrm{R}^{2}$ & 0.5375 & RMSE & $34,402.9200$ \\
\hline Adj. $\mathrm{R}^{2}$ & 0.7517 & MAE & $79,806.6800$ & Adj. $R^{2}$ & 0.4998 & MAE & $25,063.4000$ \\
\hline Akaike Info. Crit. & -1.9469 & MAPE & 6.0511 & Akaike Info. Crit. & -1.0129 & MAPE & 9.8396 \\
\hline Schwarz Info. Crit. & -1.5387 & Theil & 0.0382 & $\begin{array}{l}\text { Schwarz Info. } \\
\text { Crit. }\end{array}$ & -0.8288 & Theil & 0.0671 \\
\hline $\begin{array}{l}\text { HQ Info. Crit. } \\
\text { DW-Stat }\end{array}$ & -1.7863 & $\begin{array}{l}\text { Proporción } \\
\text { Proyección- } \\
\text { Muestra }\end{array}$ & $32 \%$ & HQ Info. Crit. & -0.9419 & $\begin{array}{l}\text { Proporción } \\
\text { Proyección- } \\
\text { Muestra }\end{array}$ & $37 \%$ \\
\hline
\end{tabular}

***Significativo al $1 \%, * *$ Significativo al $5 \%, *$ Significativo al $10 \%$ 
El Gráfico $\mathrm{N}^{\circ} 2$, presenta las proyecciones de granel sólido para las terminales APMTC y TC del periodo 2019 al 2023.

\section{Gráfico N²: APMTC y TC-Proyección de Graneles Sólidos (TM)}
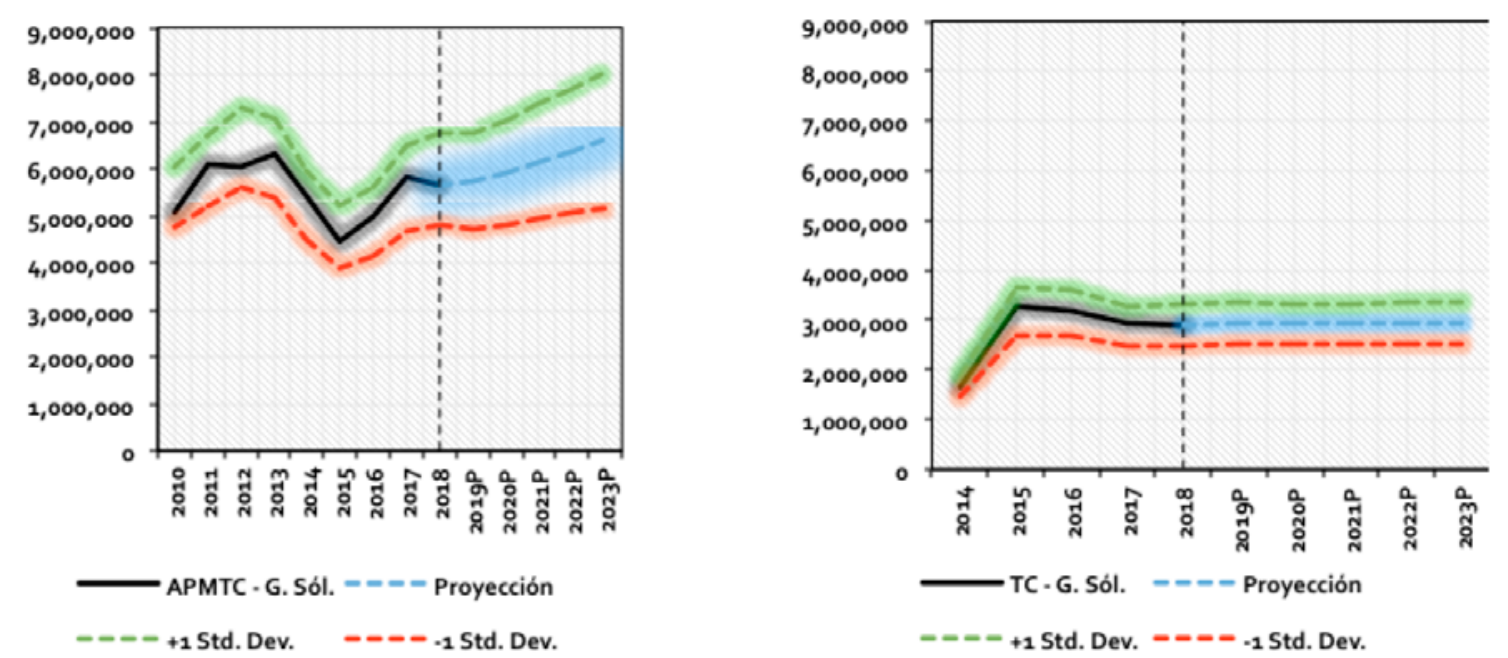

Fuente y Elaboración: Propia

La Tabla $\mathrm{N}^{\circ} 8$ presenta el resultado del mejor modelo obtenido para la carga de granel líquidos de APMTC, según lo detallado en la sección metodológica. La especificación incluye los componentes AR(1), y GARCH(1,0). Asimismo, contempla la inclusión del índice de apertura comercial y de dos variables dummy para los periodos de 2010-2011 y 2014-2015.

Tabla N²: APMTC -Estimación del Modelo de Carga de Graneles Líquidos

\begin{tabular}{|c|c|c|c|}
\hline \multicolumn{4}{|c|}{$\begin{array}{c}\text { Terminal Portuario: APMTC } \\
\text { Método: ARMA Maximum Likelihood (BFGS) } \\
\text { Variable Dependiente: DLOG(GLIQ) }\end{array}$} \\
\hline \multicolumn{2}{|c|}{ Mean Equation } & \multicolumn{2}{|c|}{ Variance Equation } \\
\hline Variable & Coeficiente / Desv. Est. & Variable & Coeficiente / Desv. Est. \\
\hline \multirow[t]{2}{*}{$\mathrm{C}$} & 0.011 & $\mathrm{C}$ & 0.001 \\
\hline & $(0.008)$ & & $(0.001)$ \\
\hline \multirow[t]{2}{*}{ DLOG(IAC(-2)) } & $-0.792 * * *$ & GARCH(-1) & $0.881 * * *$ \\
\hline & $(0.219)$ & & $(0.134)$ \\
\hline \multirow[t]{2}{*}{ DUMMY 5} & $0.709 * * *$ & & \\
\hline & $(0.036)$ & & \\
\hline \multirow[t]{2}{*}{ DUMMY 6} & $-0.065^{*}$ & & \\
\hline & $(0.038)$ & & \\
\hline \multirow[t]{2}{*}{$\mathrm{AR}(1)$} & $-0.685 * * *$ & & \\
\hline & $(0.105)$ & & \\
\hline
\end{tabular}




\begin{tabular}{cccc}
\hline Observaciones Incluidas & 60 & Periodos Proyectados & 20 \\
$\mathrm{R}^{2}$ & 0.7917 & RMSE & $59,172.7400$ \\
Adj. $\mathrm{R}^{2}$ & 0.7766 & MAE & $45,902.2300$ \\
Akaike Info. Crit. & -1.5970 & MAPE & 9.5331 \\
Schwarz Info. Crit. & -1.3527 & Theil & 0.0522 \\
HQ Info. Crit. & -1.5014 & Proporción Proyección & $33 \%$ \\
DW-Stat & 1.9860 & Muestra & \\
\hline
\end{tabular}

$* * *$ Significativo al $1 \%, * *$ Significativo al $5 \%, *$ Significativo al $10 \%$

El Gráfico $\mathrm{N}^{\circ} 3$, presenta las proyecciones de granel líquido para la terminal APMTC durante el periodo 2019-2023.

\section{Gráfico N³: APMTC-Proyección de Graneles Líquidos (TM)}

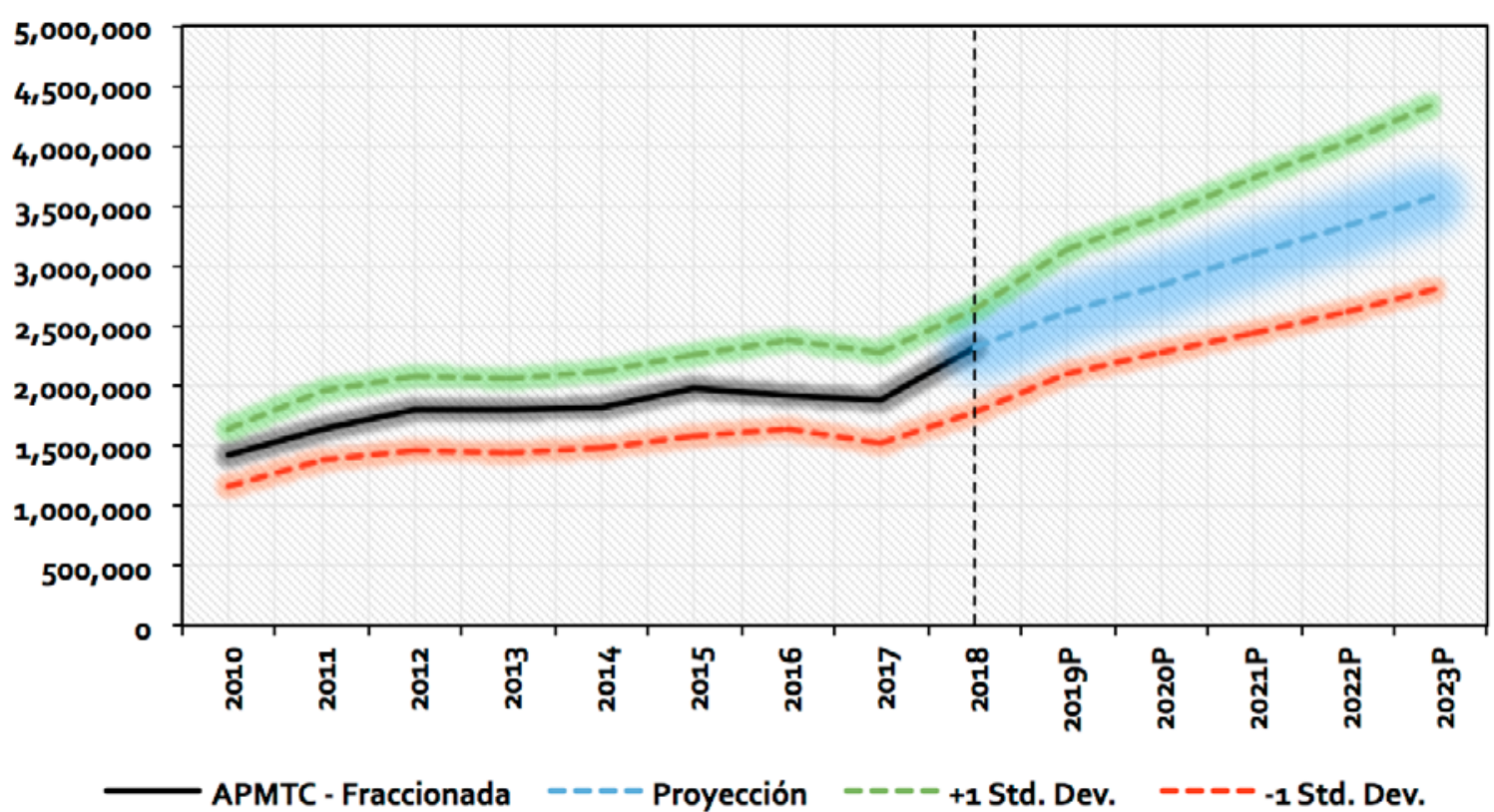

Fuente y Elaboración: Propia

La Tabla $\mathrm{N}^{\circ} 9$ presenta los resultado de los mejores modelos obtenido para la carga en contenedores de APMTC y DPWC, según lo detallado en la sección metodológica. En el caso de APMTC, la especificación incluye los componentes AR(1), SAR(18) y MA(1). Asimismo, contempla la inclusión de tres variables dummy: (i) la apertura del Terminal de Contenedores - Muelle Sur (DPWC) en el 2010, (ii) una caída de movimiento de carga para el primer trimestre del año 2012 y (iii) el alcance de la capacidad máxima del terminal portuario en el periodo 2015-2016. Respecto a las variables explicativas, se incluye la variable exportación de productos tradicionales e importación de bienes de capital.

Por su parte, para DPWC, la especificación contiene el componente AR(2), MA(1), SMA(10), la exportación de productos tradicionales, la importación de bienes de capital y dos variables Dummy. La primera por el inicio de operaciones de DPWC durante los últimos meses del año 2010 y la segunda durante el periodo 2015-2016 por el alcance de la capacidad máxima de APMTC. 
Tabla N9: APMTC y DPWC - Estimación del Modelo de Carga de Contenedores

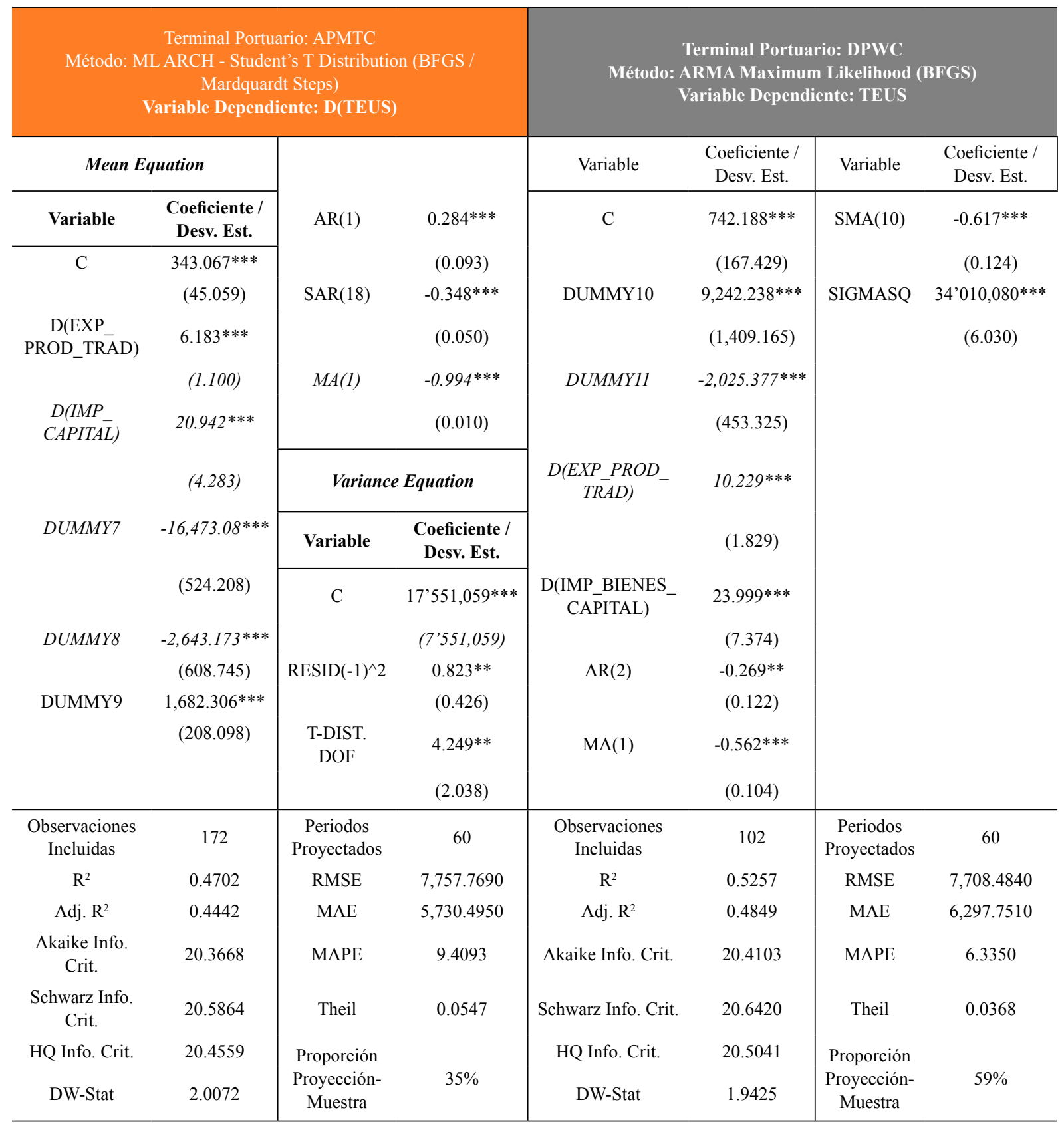

*** Significativo al 1\%, **Significativo al $5 \%, *$ Significativo al $10 \%$

El Gráfico $\mathrm{N}^{\circ} 4$, presenta las proyecciones de carga contenedorizada para las terminales APMTC y DPWC durante el periodo 2019-2023. 


\section{Gráfico N4: APMTC y DPWC-Proyección de Carga Contenedorizada} (TEU's)
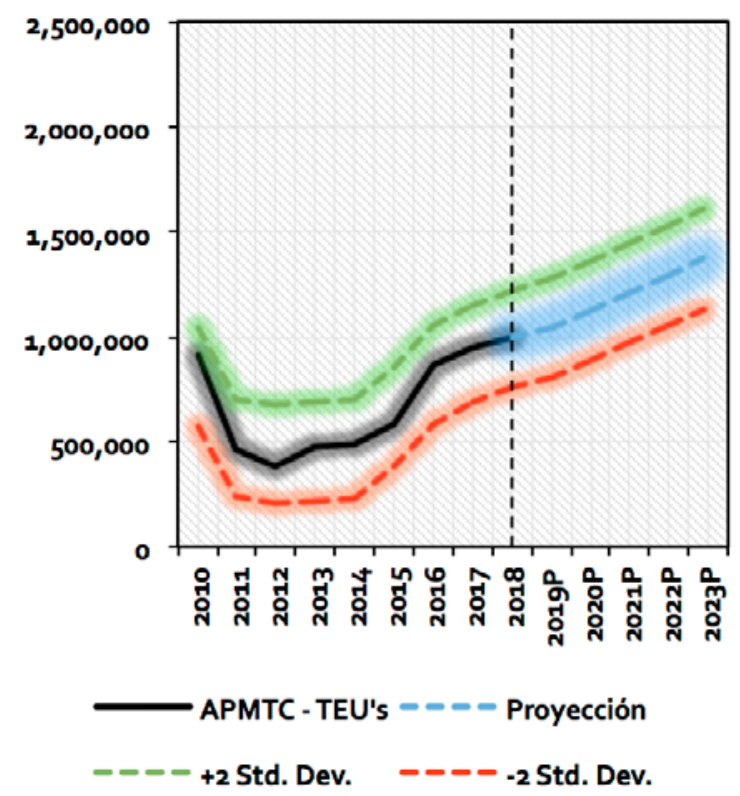

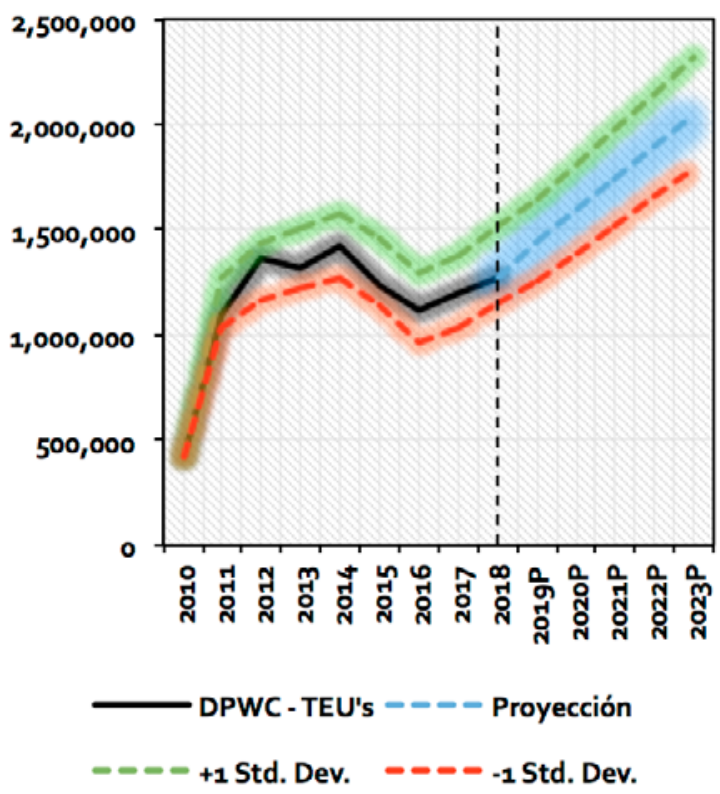

Fuente y Elaboración: Propia

La Tabla $\mathrm{N}^{\circ} 10$ presenta el resultado del mejor modelo obtenido para la carga de granel rodante de APMTC, según lo detallado en la sección metodológica. La especificación incluye los componentes AR(1), AR(2) y SAR(3). Asimismo, contempla la inclusión de la variable importación de bienes de capital y el índice de expectativas de la economía a 3 meses.

\section{Tabla N${ }^{\circ}$ 10: Especificación de Modelo de Proyección por Tipo de Carga - Carga Rodante (TM)}

\begin{tabular}{|c|c|c|c|}
\hline \multicolumn{4}{|c|}{$\begin{array}{l}\text { Terminal Portuario: APMTC } \\
\text { Método: ARMA Maximum Likelihood (BFGS) } \\
\text { Variable Dependiente: DLOG(RODANTE) }\end{array}$} \\
\hline \multirow{5}{*}{ DLOG(IMP_CAPITAL) } & Coeficiente / Desv. Est. & Variable & Coeficiente / Desv. Est. \\
\hline & 0.002 & $A R(1)$ & $-0.633 * * *$ \\
\hline & $(0.007)$ & & $(0.107)$ \\
\hline & $1.435 * * *$ & $\operatorname{AR}(2)$ & $-0.864 * * *$ \\
\hline & $(0.147)$ & & $(0.051)$ \\
\hline \multirow[t]{4}{*}{ DLOG(EXP5(-1)) } & $0.730 * * *$ & $\operatorname{SAR}(3)$ & $-0.718 * * *$ \\
\hline & $(0.141)$ & & $(0.113)$ \\
\hline & & SIGMASQ & $0.021 * * *$ \\
\hline & & & $(0.004)$ \\
\hline Observaciones Incluidas & 62 & Periodos Proyectados & 20 \\
\hline $\mathrm{R}^{2}$ & 0.6608 & RMSE & $8,742.5440$ \\
\hline Adj. $R^{2}$ & 0.6238 & MAE & 12.0820 \\
\hline Akaike Info. Crit. & -0.7262 & MAPE & 12.0820 \\
\hline Schwarz Info. Crit. & -0.4861 & Theil & 0.0853 \\
\hline HQ Info. Crit. & -0.6319 & cción-Muestra & $32 \%$ \\
\hline DW-Stat & 1.6675 & Proporcion Proyeccion-IVuestra & $32 \%$ \\
\hline
\end{tabular}

$* * *$ Significativo al $1 \%, * *$ Significativo al $5 \%, *$ Significativo al $10 \%$ 
El Gráfico $\mathrm{N}^{\circ} 5$, muestra la proyección de carga rodante para la terminal APMTC durante el periodo 2019-2023.

\section{Gráfico $\mathbf{N}^{\circ}$ 5: APMTC-Proyección de Carga Rodante (TM)}

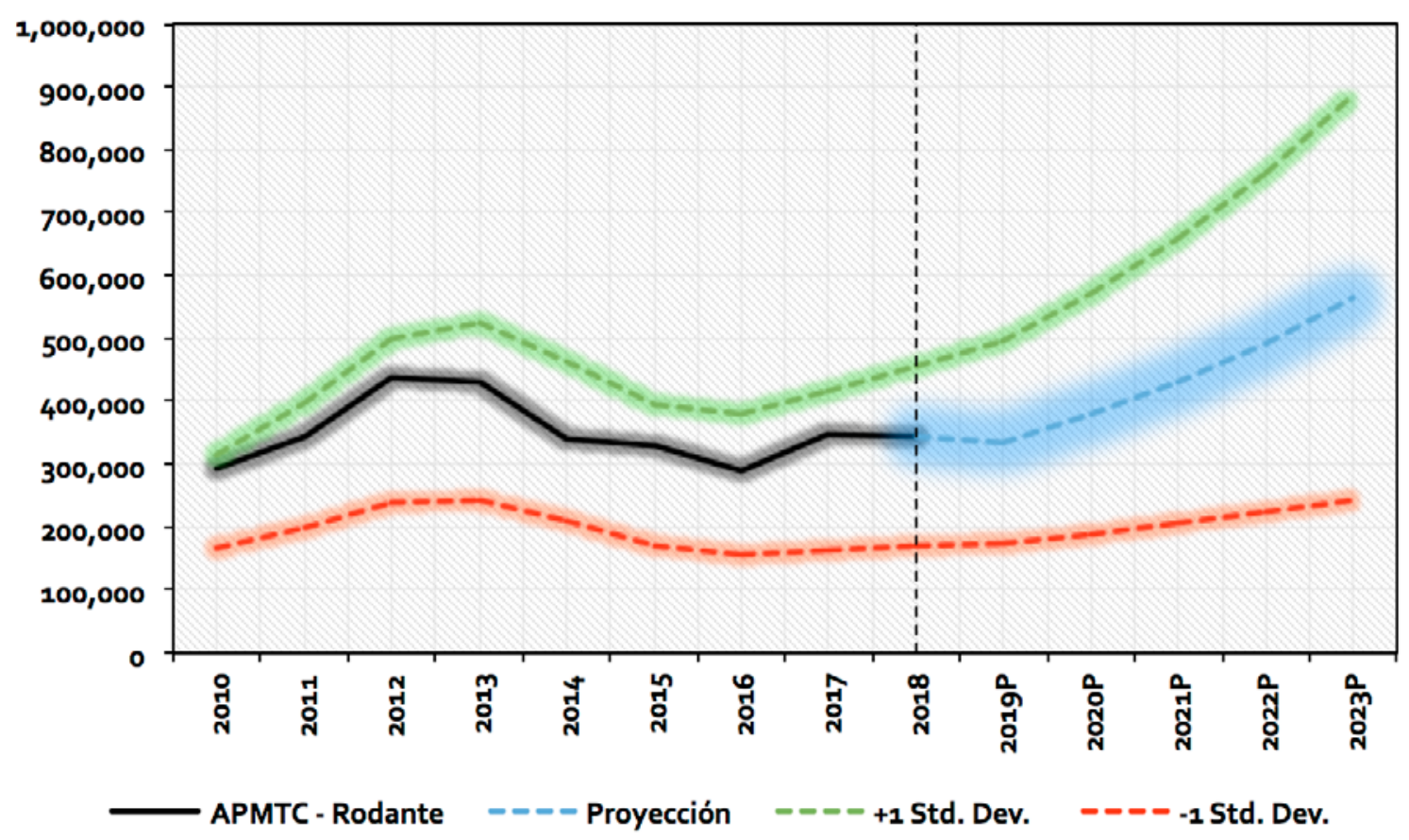

Fuente y Elaboración: Propia

\section{CONCLUSIONES}

El objetivo del artículo es estimar y proyectar la demanda de tráfico de carga para las terminales APMTC, DPWC y TC del Puerto del Callao durante el periodo 2019-2023.

Para ello, a diferencia de otros estudios, se emplean datos de frecuencia mensual, así como el de otras variables macroeconómicas relacionadas principalmente con la demanda de carga; tales como la importación de bienes de capital, la importación de bienes de consumo, la exportación de productos tradicionales, el índice de expectativas de la economía a 3 meses, el índice de apertura comercial y el precio del cobre. Al respecto, la metodología a emplear es la de análisis de series de tiempo SARIMAX. Esto debido a que dicha metodología permite un mejor tratamiento de los datos mensuales para recoger patrones estacionales, así como el poder incluir variables explicativas al modelo.
Los resultados muestran que el modelo SARIMAX presenta un buen ajuste para recoger el proceso generador de datos del tráfico de carga mensual de las terminales del puerto del Callao.

De esta manera, para APMTC, se proyecta que al 2023 movilice aproximadamente 3.6 millones de TM de carga fraccionada, 6.4 millones de TM de granel sólido, 3.3 millones de TM de granel líquido, 1.38 millones de Contenedores (TEU's) y 564 mil TM de carga rodante.

Así, las inversiones establecidas en el contrato de concesión suscrito entre APMTC y el Estado Peruano generarían que se activen los gatillos de inversión de las etapas 3 y 4 de acuerdo a lo establecido por el contrato de concesión.

Por su parte, en el caso de DPWC, se espera que para el año 2023 se movilicen aproximadamente un total de 2 millones de TEU's. Esto implica 
que el terminal activaría gatillos de inversión de las sub-fases $2 \mathrm{~B}$ y $2 \mathrm{C}$ de acuerdo a lo establecido por el contrato de concesión.

Para el caso de TC se espera que para el año 2023, de acuerdo a las proyecciones realizadas, se movilicen aproximadamente un total de 3.3 millones de TM de graneles sólidos.

En ese sentido, se espera que para el año 2023 se movilicen un total de 17 millones de TM entre los tres terminales del Puerto del Callao y 3.4 millones de TEU's entre DPWC y APMTC.

Por lo tanto la metodología SARIMAX, empleada en la presente investigación, podría proporcionar al regulador de mayores herramientas para evaluar metodologías prospectivas para las revisiones tarifarias, las cuales les permita incluir las proyecciones de demanda de tráfico en el Puerto del Callao. Asimismo, para mejorar la realización de inversión en infraestructura portuaria por parte de los concesionarios y de los distintos actores involucrados en el sector portuario como las compañías navieras, autoridades portuarias, operadores y otros inversionistas.

\section{REFERENCIAS}

[1] Abdelali Hajbi (2011) Traffic Forecasting in Moroccan Ports, Supply Chain Forum: An International Journal, 12:4, 26-35.

[2] Bollerslev, Tim (1986). Generalized Autoregressive Conditional Heteroskedasticity. Journal of Econometrics, 307-327.

[3] De Rus, G., Campos, J. y Nombela, G. (2003) Economía del transporte, Antoni Bosch Editor.

[4] Dragan, Dejan; Kramberger, Tomaz; Intihar, Marko. (2014). A comparison of Methods for Forecasting the Container Throughput in North Adriatic Ports.

[5] Dragan, Dejan; Kramberger, Tomaz; Intihar, Marko. (2017). Container Throughput Forecasting Using Dynamic Factor Analysis and ARIMAX Model. Promet - Traffic \& Transportation, Vol. 29, No. 5, 529-542.

[6] Enders, W. (2014). Applied Econometric Time Series. en W. Enders, Applied Econometric Time Series (pp. 76-77). Tuscaloosa, Alabama: Wiley \& Sons, Inc.
[7] Hui, Eddie; Seabrook, William; Wong, Gordon (2004). Forecasting Cargo Throughput for the Port of Hong Kong: Error Correction Model Approach. Journal of Urban Planning and Development, 195203.

[8] Kotcharat, Pitinoot, A forecasting model for container throughput: empirical research for Laem Chabang Port, Thailand (2016). World Maritime University Dissertations. 538.

[9] Mohamed, Yasmine R. (2016). Container Throughput Modelling and Forecasting: An Empirical Dynamic Econometric Time Series Approach. Amberes: University of Antwerp - Faculty of Applied Economics.

[10] Wen-Yi Peng, Ching-Wu Chu (2009). A Comparison of univariate methods for forecasting container throughput volumes. Department of shipping and Transportation Management, National Taiwan Ocean University.

[11] Veerachi Gosasang, Watcharavee Chandraprakaikul, Supaporn Kiattisin (2011). A Comparison of Traditional and Neural Networks Forecasting Techniques for Container Throughput at Bangkok Port. The Asian Journal of Shipping and Logistics.

[12] Gamassa Pascal Kany Prud'ome, Chen Yan (2017). Application of several models for the forecasting of the container Throughput of the Abidjan in the Republic of Côte d'Ivoire. International Journal of Engineering Research in Africa.

[13] Shih-Huang Chen, Jun-Nan Chen (2010). Forecasting container throughputs at ports using genetic programming. Dept. Transportation Technology and Management, Feng-Chia University, Taiwan.

[14] Peter M. Schulze, Alexander Prinz (2014). Forecasting container transshipment in Germany. Institute of Statistics and Econometrics, Johannes Gutenberg University, Mainz, Germany. 